\title{
Radioisotope Distribution Program Progress Report for January 1977
}

\author{
E. Lamb
}

\section{OAK RIDGE NATIONAL LABORATORY}




\section{DISCLAIMER}

This report was prepared as an account of work sponsored by an agency of the United States Government. Neither the United States Government nor any agency Thereof, nor any of their employees, makes any warranty, express or implied, or assumes any legal liability or responsibility for the accuracy, completeness, or usefulness of any information, apparatus, product, or process disclosed, or represents that its use would not infringe privately owned rights. Reference herein to any specific commercial product, process, or service by trade name, trademark, manufacturer, or otherwise does not necessarily constitute or imply its endorsement, recommendation, or favoring by the United States Government or any agency thereof. The views and opinions of authors expressed herein do not necessarily state or reflect those of the United States Government or any agency thereof. 


\section{DISCLAIMER}

Portions of this document may be illegible in electronic image products. Images are produced from the best available original document. 
This report was prepared as an account of work sponsored by the United States Guvernment. Neither the United States nor the Energy Research and Development Administration/United States Nuclear Regulatory Commission, nor any of their employees, nor any of their contractors, subcontractors, or their employees, makes any warranty, express or implied, or assumes any legal liability or responsibility for the accuracy, completeness or usefulness of any information, apparatus, product or process disclosed, or represents that its use would not infringe privately owned rights. 
Contract No. W-7405-eng-26

OPERATIONS DIVISION

\title{
RADIOISOTOPE DISTRIBUTION PROGRAI
} PROGRESS REPORT FOR JANUARY 1977

\author{
E. Lamb \\ Work Sponsored by \\ ERDA Division of Biomedical and \\ Environmenta1 Research \\ Date Published - March - 1977
}

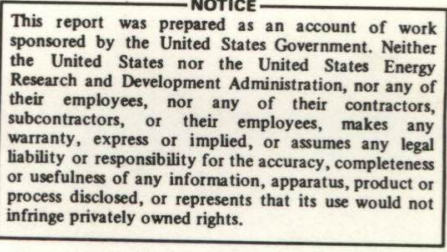

NOTICE This document contains information of a preliminary nature. It is subject to revision or correction and therefore does not represent a final report.

OAK RIDGE NATIONAL LABORATORY

Oak Ridge, Tennessee 37830

operated by

UNION CARBIDE CORPORATION

for the

ENERGY RESEARCH AND DEVELOPMENT ADMINISTRATION 
THIS PAGE

\section{WAS INTENTIONALLY LEFT BLANK}


CONTENTS

$\underline{\text { Page }}$

RADIOISOTOPE PRODUCTION AND MATERIALS DEVELOPMENT. . . . . . 1

REACTOR-PRODUCED RADIOISOTOPES . . . . . . . . . . . I 1

Reactor Products Pilot Production. . . . . . . . 1

ACCELERATOR-PRODUCED ISOTOPES. . . . . . . . . . . 1

Cyclotron Products Pilot Production. . . . . . . . . 1

FISSION PRODUCTS . . . . . . . . . . . . . . 2

Krypton-85 Enrichment Facility . . . . . . . . . 2

Cesium-137 Pilot Production. . . . . . . . . . . 2

Strontium-9.0 Pilot Production. . . . . . . . . . 3

Short-Lived Fission Production ........... . 4

RADIOISOTOPE SALES . . . . . . . . . . . . . . . 4 4

PUBLICATIONS . . . . . . . . . . . . . . . . 5

REPORTS. . . . . . . . . . . . . . . . . . 5 
RADIOISOTOPE DISTRIBUTION PROGRAII

PROGRESS REPORT FOR JAIIUARY 1977

E. Lamb

RADIOISOTOPE PRODUCTION AND MATERIALS DEVELOPIIENT

REACTOR-PRODUCED RADIOISOTOPES

Reactor Products Pilot Production (R. W. Schaich)

(Production and Inventory Accounts)

$\frac{\text { Processed Units }}{\text { Radioisotope }} \frac{\text { Amount (mCi) }}{21}$

ACCELERATOR-PRODUCED ISOTOPES

Cyclotron Products Pilot Production (M.R. Skidmore)

(Production and Inventory Accounts)

January 1977 ORIJ 86-Inch Cyclotron runs for ORNL and non-ORNL programs are given in Table 1 .

Table 1. Cyclotron Irradiations and Runs for January 1977

\begin{tabular}{llc}
\hline Date & Product & Total Time Total \\
(hr:min) Charges
\end{tabular}

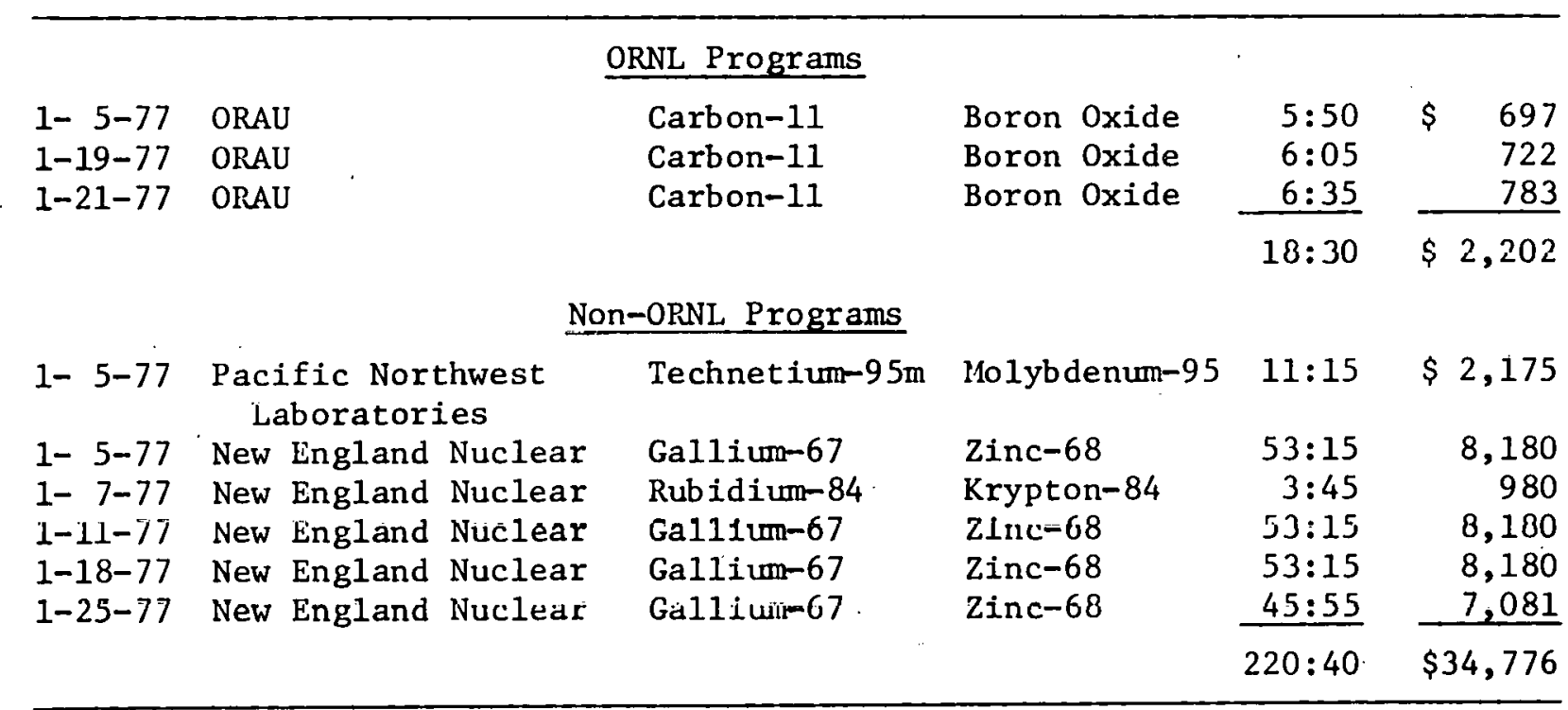




\section{Cyclotron Operations}

The cyclotron operated 239 hours during the month of January. Operations were interrupted three times during the month for equipment repairs. On January 2, 1977 a 440 volt A.C. terminal in cubicle 96 had to be repaired. On January 9, 1977 a vacuum failure occurred due to a bad bearing on an electric motor on a Kinney pump. The electric motor was replaced, but it too had a bearing failure within 15 minutes of startup because the belts had been tightened too tight when the motor was installed. On January 12 th the heating element on the east 20-inch diffusion pump was replaced. The cyclotron did not operate from January 25 th through January 31 st due to RF problems. Two carbon plates on the liner were found to be cracked, the bias bushing was leaking badly, and the deflector had a leak in the flexible cooling water line inside the vacuum tank.

FISSION PRODUCTS

Krypton-85 Enrichment Facility (R. W. Schaich)

The ${ }^{85} \mathrm{Kr}$ enrichment columns operated satisfactorily during the month of January. A maintenance program was initiated in January to upgrade the operational safety of the system. This maintenance should be completed by the middle of February on $50 \%$ of the columns. At that time the remaining sections will be unloaded and a material balance for the run completed.

Cesiurn-137 Pilot Production (R. W. Schaich)

(Production and Inventory Accounts)

1. Process Status

Process equipment is in standby status.

2. Operational Summary

\section{Product Inventory}

(Decay calculated through August 31, 1976)

Inventory Material

Amount (Ci)

Cesiurn-137 chloride powder

34,900

Total Inventory Material

34,900 


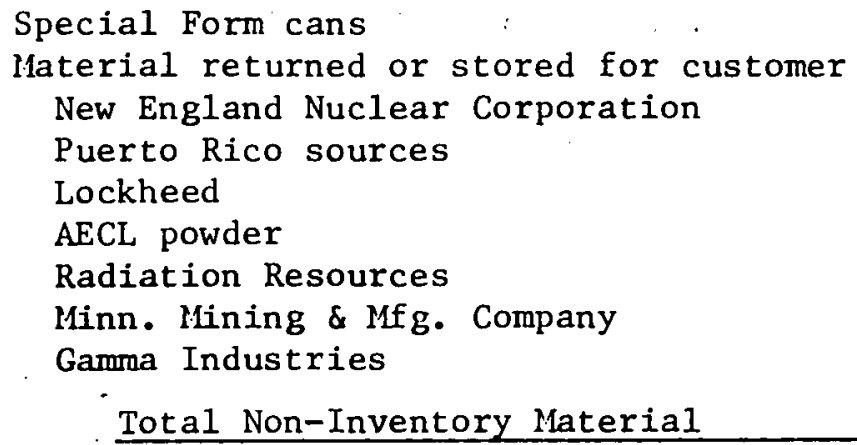

Total Non-Inventory Material

TOTAL INVENTORY AND NON-IIVENTORY IATERIAL

\section{Fabrication Summary}

\begin{tabular}{|c|c|c|c|c|c|c|}
\hline \multirow{2}{*}{ 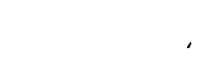 } & \multicolumn{2}{|c|}{ Jan. 1977} & \multicolumn{2}{|c|}{ CY 1977} & \multicolumn{2}{|c|}{ FY 1977} \\
\hline & No. & $\mathrm{Ci}$ & No. & $\mathrm{Ci}$ & No. & $\mathrm{Ci}$ \\
\hline \multicolumn{7}{|l|}{ Sources } \\
\hline Fabricated & 5 & 15,846 & 5 & 15,846 & 22 & 16,146 \\
\hline Shipped & 5 & 15,846 & 5 & 15,846 & 64 & 30,786 \\
\hline \multicolumn{7}{|c|}{ Special Form Cans } \\
\hline Fabricated & 0 & 0 & 0 & 0 & 0 & 0 \\
\hline Shipped & 2 & 1,900 & 2 & 1,900 & 2 & 1,900 \\
\hline
\end{tabular}

\section{Current Orders}

All orders on hand have been completed and the material placed into storage awaiting receipt of release for the material.

Strontium-90 Pilot Production (R. W. Schaich) (Production and Inventory Accounts)

\section{Process Status}

The 20,000 curie ${ }^{90} \mathrm{Sr}$ heat source that was fabricated in November, 1976 for SNAM Progetti, Italy will be encapsulated and shipped during February, 1977. Weld penetration specifications have been resolved with the customer. 


\section{Product Inventory}

(Decay calculated through Augusl 31, 1976)

Inventory Material

${ }^{90} \mathrm{Sr}$ titanate powder $( \pm 5 \%)$

Sources in fabrication

RCA source

${ }^{90} \mathrm{Sr}$ silicate powder (est.)

Stock powder cans

Total Inventory Material
Amount (Ci)

77,000

20,000

57,300

28,000

3,790

186,090

Non-Inventory Material

Amount (Ci)

Calorimeter Standards

FPDL recovery material

4,900

19,700

44,000

Quehanna recovery material

11,700

Weather Bureau source

SNAP-7B

SNAF $-7 C$

160,200

25,200

146,600

SNAP material purchase $\mathrm{a}^{\mathrm{a}}$

$\underline{254,500}$

666,800

TOTAL INVENTORY AND NON-INVENTORY MATERIAL

852,890

${ }^{2}$ Strontium-90 purchased under DRRD program.

Fabrication Summary

$$
\frac{\text { Jan. } 1977}{\text { No. } \frac{\text { C1 }}{}}
$$

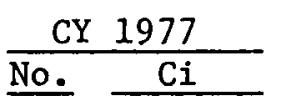

$\frac{\text { FY } 1977}{\text { No. } \quad \text { Ci }}$

Sources
Fabricated
Shipped

Special Form Cans

Fabricated

Shipped

$\begin{array}{llllll}0 & 0 & 0 & 0 & 0 & 0 \\ 0 & 0 & 0 & 0 & 0 & 0\end{array}$

$\begin{array}{lll}0 & 0 & 0 \\ 0 & 0 & 0\end{array}$

0
Amount (Ci)

1100

40

14

16

39 


\section{RADIOISOTOPE SALES}

J. E. Ratzedge

Shipments made during the month that may be of interest are listed below:

Customer

Isotope

$\underline{\text { Amount }}$

Large Quantities

Schwarz/Mann

American Atomics

ICN Pharmaceuticals

New England Nuclear Corporation

Saunders-Roe, England
Tritium
Tritium
Tritium
Tritium
Tritium

$1,000 \mathrm{Ci}$

$6,000 \mathrm{Ci}$

$1,000 \mathrm{Ci}$

4,000 Ci

$10,000 \mathrm{Ci}$

Withdrawn Items

Cleveland Metropolitan General Hospital Iodine-131

$50 \mathrm{mCi}$

Items Used in Cooperative Programs

University of Southern California

Platinum-195m

$12 \mathrm{mCi}$

George Washington University Medical Ctr. Platinum-195m

$30 \mathrm{mCi}$

The radioisotope sales and shipments for October 1975 through January 1976 and the first four months of fiscal year 1977 are given in Table 2.

Table 2. Radioisotope Sales and Shipments

\begin{tabular}{|c|c|c|c|c|}
\hline Item & \multicolumn{2}{|c|}{$\begin{array}{c}10-1-75 \text { thru } \\
1-31-76\end{array}$} & \multicolumn{2}{|c|}{$\begin{array}{c}10-1-76 \text { thru } \\
1-31-77\end{array}$} \\
\hline $\begin{array}{l}\text { Inventory items } \\
\text { Major products } \\
\text { Radioisotope services } \\
\text { Cyclotron Irradiations } \\
\text { liscellaneous processed materials } \\
\text { Packing and Shipping }\end{array}$ & $\$$ & $\begin{array}{r}103,410 \\
15,767 \\
31,859 \\
54,849 \\
19,288 \\
34,715 \\
\end{array}$ & $\$$ & $\begin{array}{r}102,985 \\
36,399 \\
61,507 \\
165,934 \\
18,502 \\
63,189 \\
\end{array}$ \\
\hline Total & $\$$ & 259,888 & $\$$ & 448,516 \\
\hline Number of shipments & & 804 & & 792 \\
\hline
\end{tabular}

PUBLICATIONS

REPORTS

E. Lamb, Radioisotope Distribution Program Progress Report for December 1976, ORNL/TM-5791, Oak Ridge National Laboratory (January 1977). 
THIS PAGE

WAS INTENTIONALLY

LEFT BLANK 


\section{INTERNAL DISTRIBUTION}

ORNL/TM-5845

1. E. E. Beauchamp

2. T. A. Butler

3. F. N. Case

4. W. R. Casto

5. J. A. Cox

6. R. F. Hibbs.

7. E. Lamb

8. H. H. Nichol

9. C. L. Ottinger

10. J. K. Poggenburg

11. H. Postma
12. H. E. Ramsey

13. J. E. Ratledge

14. C. R. Richmond

15. R. W. Schaich

16. M. R. Skidmore

17. . M. J. Skinner

18-19. Central Research Library

20-21. Laboratory Records Department

22. Laboratory Records - RC

23. Document Reference Section

\section{EXTERNAL DISTRIBUTION}

24. B. J. Dropesky, LASL, Los Alamos, New Mexico

25-26. J. H. Jarrett, PNL, Richland, Washington

27. D. K. Jones, Richland Operations Office, Richland, Washington

28. J. N. Maddox, ERDA-DBER, Washington, D.C.

29. H. A. O'Brien, LASL, Los Alamos, New Mexico

30. F. J. Skozen (Krizek), Argonne Cancer Research Hospital, Chicago

31. L. G. Stang, Jr., BNL, New York

32. W. H. Weyzen, ERDA-DBER, Washington, D.C.

33-34. R. W. Wood, ERDA-DBER, Washington, D.C.

35. Donner Laboratory Library, Univ. of California, Berkeley, Calif., 94720

36. Research and Technical Support Division, ORO

37-38. Technical Information Center 